\title{
EVALUATION OF EXTENDED SPECTRUM $\beta$-LACTAMASE (ESBL) IN URINARY ISOLATES OF ESCHERICHIA COLI AND KLEBSIELLA PNEUMONIAE
}

\author{
Manish Purohit ${ }^{1}$, Anita Mutha ${ }^{2}$
}

${ }^{1}$ Assistant Professor, Department of Microbiology, MGM Medical College, Indore.

2 Professor \& Head, Department of Microbiology, MGM Medical College, Indore.

\begin{abstract}
Urinary tract infection are most prevalent infection worldwide, Escherichia coli and Klebsiella pneumoniae are commonly implicated in causing such infection. These bacteria produce large amount of ESBLs and make treatment difficult with betalactam group of antibiotics. ESBLs producing bacteria also show resistance to other group of antibiotics, hence knowledge of ESBL producing bacteria and their susceptibility pattern is helpful in selection of appropriate antibiotic for treatment of UTI patient. Current study was aimed to evaluate presence of ESBL producing E. coli and Klebsiella pneumoniae from urinary isolates and their susceptibility pattern.
\end{abstract}

\section{METHODS}

Between October 2014 to December 2014, 725 Urine specimens were received in the Department of Microbiology, Tertiary Care Centre of Central India. The samples were cultured on UTI chrome agar (Hi-Media) with the standard calibrated loop (Diameter $0.04 \mathrm{~mm})$ by semi-quantitative method and the isolates with significant bacteriuria $\left(\geq 10^{5} \mathrm{CFU} / \mathrm{ml}\right)$ were included in study. Antimicrobial susceptibility test was carried out using various antimicrobial discs by Kirby Bauer disc diffusion method as per the

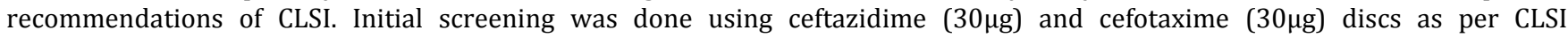
recommendation.

Confirmation of ESBL production was done by Phenotypic Confirmatory Disc Diffusion Test (PCDDT) using ceftazidime (30 $\mu \mathrm{g})$ and ceftazidime + clavulanic acid $(30 \mu \mathrm{g}+10 \mathrm{ug})$ disc as per guidelines of CLSI (2014).

\section{RESULT}

Out of a total of 725 urine specimens investigated for significant bacteriuria, 93 (12.82\%) E. coli and 28 (3.8\%) K. pneumoniae were isolated. Initial screening revealed $74(79.56 \%)$ isolates of E.coli and $18(64.28 \%)$ isolates of K. pneumoniae as probable ESBL producers. Further testing by PCDDT method confirmed 29 (31.18\%) of E. coli and 5 (17.85\%) of K. pneumoniae isolates as ESBL producers, making a total of $34(36.95 \%)$. These ESBL producing uropathogens showed maximum resistance to cotrimoxazole $(100 \%)$ and maximum sensitivity to carbapenem group of antibiotics $(100 \%)$.

\section{CONCLUSION}

Our study showed emergence and occurrence of ESBL producing E.coli and Klebsiella pneumoniae in urinary isolates. $100 \%$ sensitivity to carbapenem group of antibiotics was found among ESBL producer advocate there use in UTI patients. Monitoring of ESBL production and antimicrobial susceptibility testing are necessary to avoid treatment failure in patients with UTI.

\section{KEYWORDS}

ESBL, E. coli, K. pneumoniae.

HOW TO CITE THIS ARTICLE: Purohit M, Mutha A. Evaluation of extended spectrum $\beta$-lactamase (ESBL) in urinary isolates of escherichia coli and klebsiella pneumoniae. J Evolution Med Dent Sci 2016;5(1):19-22, DOI: 10.14260/jemds/2016/5

\section{INTRODUCTION}

The emergence of resistance to betalactam antibiotics due to production of betalactamases in the past two decades has resulted in a major clinical crisis. ${ }^{1}$ ESBL is a $\beta$-lactamase first identified in the 1980s and have gradually spread throughout the world by nosocomial routes. ESBLs are chromosomal or plasmid mediated $\beta$-lactamases (Enzymes that cleave the $\beta$ lactam ring), which have mutated from pre-existing broadspectrum $\beta$-lactamases (TEM-1, TEM-2, SHV-1) as a consequence of widespread use of $3^{\text {rd }}$ generation Cephalosporins as well as Aztreonam. ESBL-producing genes are normally harboured on plasmids $80 \mathrm{~kb}$ in size or larger and most often carry resistance determinants for aminoglycosides,

Financial or Other, Competing Interest: None.

Submission 14-12-2015, Peer Review 15-12-2015,

Acceptance 30-12-2015, Published 01-01-2016.

Corresponding Author:

Dr. Manish Purohit,

Assistant Professor

Department of Microbiology,

MGM Medical College,

Indore.

E-mail: manishpurohit752gmail.com

DOI:10.14260/jemds/2016/5 fluoroquinolones, tetracyclines, Chloramphenicol and Cotrimoxazole, making the micro-organisms resist a wide variety of drugs. ${ }^{2}$ These plasmids are transferable from one bacterial strain to the next and between different bacterial species. $^{3} \quad$ ESBL producing organisms are distributed worldwide and their prevalence is increasing. There is a rising incidence of Urinary Tract Infection (UTI) with ESBL producing bacteria. ${ }^{4}$ Escherichia coli and Klebsiella pneumoniae are the major bacterial pathogens being isolated and reported from Mid-Stream Urine (MSU) specimens, globally. These uropathogens are mostly implicated as the major Extended Spectrum $\beta$-Lactamase (ESBL) producers, severely limiting the therapeutic management in cases of urinary tract infections. ${ }^{3}$

Further there have been significant changes in the antimicrobial resistance patterns among Extended Spectrum $\beta$-Lactamase (ESBL) producing pathogens makes empirical treatment of these infections difficult. Antibiotic resistance varies according to geographic locations and is directly proportional to the use and/or misuse of antibiotics. ${ }^{5}$ Hence ESBLs are clinically significant and when detected, indicate the need for the use of appropriate antibacterial agents. ${ }^{3}$ 
This study was designed to evaluate the ESBL production among E.coli and Klebsiella pneumoniae in urinary isolates in a Tertiary Care Centre at Indore.

\section{MATERIAL AND METHODS \\ Setting}

This study was carried out in the Department of Microbiology, Mahatma Gandhi Memorial Medical College, Indore (MP). A total of 725 mid-stream urine specimen collected in a sterile container were received over a period of three months (October 2014 to December 2014), processed by semi quantitative culture technique using a standard calibrated loop (diameter $0.04 \mathrm{~mm}$ ) on UTI Chrome agar (HiMedia). After $24 \mathrm{hr}$ of aerobic incubation at $37^{\circ} \mathrm{C}$, culture growth showing significant bacteriuria were included in the study for further processing. The isolates were identified on the basis of colour produced on chrome agar as per manufacturer instructions. Antimicrobial susceptibility was determined by Kirby-Bauer disk diffusion method as per CLSI

Recommendations. ${ }^{6}$ Antimicrobial discs used were Ampicillin (10 $\mu \mathrm{g})$, Amoxycillin-Clavulanic acid (10/10), Ciprofloxacin $(10 \mu \mathrm{g})$, Levofloxacin $(5 \mu \mathrm{g})$ Cephotaxime $(30 \mu \mathrm{g})$, Ceftriaxone $(30 \mu \mathrm{g})$, Ceftazidime $(30 \mu \mathrm{g})$, Amikacin $(30 \mu \mathrm{g})$, Nitrofurantoin (10 $\mu \mathrm{g}), \quad$ Trimethoprim-sulfamethoxazole $(1.25 / 23.75 \mu \mathrm{g})$, Imipenem $(10 \mu \mathrm{g})$ and Meropenem $(10 \mu \mathrm{g})$.

\section{Screening of ESBL Production}

Ceftazidime $(30 \mu \mathrm{g})$ and Cefotaxime $(30 \mu \mathrm{g})$ discs were used as a screening agents as per CLSI guideline. Isolates showing inhibition zone size of $\leq 22 \mathrm{~mm}$ with Ceftazidime $(30 \mu \mathrm{g})$, and $\leq$ $27 \mathrm{~mm}$ with Cefotaxime $(30 \mu \mathrm{g})$ were identified as potential ESBL producers and shortlisted for confirmation of ESBL production. Klebsiella pneumoniae ATCC70063 and Escherichia coli ATCC 25922 were used as positive and negative control respectively.

\section{Phenotypic Confirmatory Disc Diffusion Test (PCDDT) for ESBL as per CLSI Recommendation \\ Procedure}

For this test a disc of Ceftazidime $(30 \mu \mathrm{g})$ alone and a disc of Ceftazidime + Clavulanic acid $(30 \mu \mathrm{g} / 10 \mu \mathrm{g})$ were used. Both the discs were placed at least $25 \mathrm{~mm}$ apart, center to center, on a lawn culture of the test isolate on Mueller Hinton Agar (MHA) plate and incubated overnight at $37^{\circ} \mathrm{C}$. Difference in zone diameters with and without clavulanic acid was measured.

\section{Interpretation}

When there is an increase of $\geq 5 \mathrm{~mm}$ inhibition zone diameter around combination disk of Ceftazidime + Clavulanic acid versus the inhibition zone diameter around Ceftazidime disk alone, it confirms ESBL production. (Figure 1).

\section{RESULTS}

A total of 725 urine specimens were processed out of this 93 (12.82\%) Escherichia coli and 28 (3.86\%) Klebsiella pneumoniae were isolated.

By the screening test, 74 (79.56\%) isolates of E. coli and $18(64.28 \%)$ isolates of Klebsiella pneumoniae were short listed as potential ESBL producers. Phenotypic confirmatory test, on screening test positive isolates detected 34 ESBL producing isolates, $29(31.18 \%)$ E. coli and $5(17.85)$ Klebsiella pneumoniae.
Among these 34, ESBL producers antibiogram revealed maximum sensitivity towards Carbapenem group of antibiotics viz. imipenem 34 (100\%) and meropenem 34 $(100 \%)$ and maximum resistance to Cotrimoxazole 34 (100\%). Resistance to ciprofloxacin and levofloxacin was 25 (73.52\%) and 24 (70.58\%) respectively. Resistance shown by Amikacin, Gentamycin and Nitrofurantoin was 7 (20\%), 7 (20\%) and $18(52.94 \%)$ respectively (Figure 2$)$.

\section{DISCUSSION}

In our country prevalence of ESBL producers have been reported since $1990 .^{2}$

Previous studies from India have reported ESBL production varying from $28 \%$ to $84 \% .^{7}$ ESBLs have also been documented in Israel, Saudi Arabia, and a variety of North African countries.8-10 The occurrence of ESBL producer in urinary isolates of E.coli and Klebsiella pneumoniae in our study was $31.18 \%$ and $17.85 \%$, respectively. Studies from different part of India reported 32\%, 41\%, 31.6\% and $24.7 \%$ ESBL positivity among urinary isolates of E.coli and these figures correlate with figures of our study.11-14 Other Indian studies reported a higher prevalence of ESBL producing strains of Klebsiella spp. $37 \%$ by Shobha et al. ${ }^{11} 38.5 \%$ by Khurana et al. ${ }^{14} 40 \%$ by S Baby Padmini et al. ${ }^{12}$ and $80 \%$ by Mathur et al. ${ }^{15}$ which is not found in our study. ESBL prevalence varies not only in different countries but also varies from hospital to hospital. ${ }^{16}$ ESBL prevalence of $44.5 \%$, $58 \%$ and $39.5 \%, 50.4 \%$ and $25-40 \%$ has been reported in $\mathrm{E}$. coli from Iran, India and Bangladesh, Nigeria and China respectively. $17,18,19,5$

Generally, pathogens in hospitals are resistant to multiple antibiotics due to increased selection pressure of antibiotics. ${ }^{20}$ Multiple drug resistance may be due to plasmids harboring several resistance genes, which are transferred from one bacterium to another. ${ }^{21}$ Such transferable plasmids also carry resistant determinants to other antimicrobial agents. Hence multidrug resistance is expected to be more common in ESBL producing organisms. ${ }^{22}$ In the present study, $61 \%$ ESBL producers showed resistance to third generation cephalosporins coexist with resistance to two or more antibiotics like ampicillin, cotrimoxazole, nitrofurantoin, ciprofloxacin and levofloxacin as also reported by Subha et al. ${ }^{23}$ and Duttaroy et al. ${ }^{24}$ indicating multidrug resistance pattern among ESBL producers. We found $100 \%$ sensitivity for carbapenem group of antibiotics and $80 \%$ sensitivity for both Amikacin and Gentamycin against ESBL producers, so these antibiotics can be used for empirical treatment. In this study all the ESBL positive isolates were found to be resistant to ampicillin and cotrimoxazole and sensitive to imipenem and meropenem, which advocates the usage of carbapenem antibiotics as the therapeutic alternative to $\beta$-lactam antibiotics as indicated in many previous studies. ${ }^{25}$

\section{CONCLUSION}

Our study showed emergence and occurrence of ESBL producing E.coli and Klebsiella pneumoniae isolates in urinary specimens. The situation is worsened due to coexistent resistance of non $\beta$-lactam group of antimicrobial agent. Hence ESBL detection and its antimicrobial sensitivity pattern should be routinely analysed to enable clinician to select most appropriate agent. 
$100 \%$ sensitivity to carbapenem group of antibiotics were found among ESBL producer advocate there use in UTI patients.

\section{REFERENCES}

1. Rugved Kulkarni, Vaishali Dohe (Kongre), Dnyaneshwari Ghadge, et al. A study of Extended Spectrum Betalactamase (ESBL) producers in clinical isolates. Medical Journal of Western India February 2013; 41(1):18-22.

2. Aruna* K, Mobashshera T. Prevalence of extended spectrum beta-lactamase production among uropathogens in South Mumbai and its antibiogram pattern. Excli Journal 2012; 11:363-372.

3. Anil Chander* and Chandrika Devi Shrestha. Prevalence of extended spectrum beta-lactamase producing Escherichia coli and Klebsiella pneumoniae urinary isolates in a Tertiary Care Hospital in Kathmandu, Nepal. Chander and Shrestha BMC Research Notes 2013; 6:487.

4. Faisal Iqbal Afridi1, Badar Jahan Farooqi1 and Arif Hussain2. Frequency of extended spectrum beta lactamase producing enterobacteriaceae among urinary pathogen isolates. Journal of the College of Physicians and Surgeons Pakistan 2011; Vol. 21 (12): 741-744.

5. Nwosu1* L, Amadi2 ES, Nwanyanwu2 CE, et al. The prevalence of extended spectrum beta-lactamases (ESBLs) among Escherichia coli and Klebsiella species urinary isolates from Abia State University Teaching Hospital (ABSUTH) aba, Abia State, Nigeria. International Journal of Microbiology and Mycology | IJMM pISSN: 2309-4796.

6. Clinical and Laboratory Standards Institute (CLSI). Performance standards for antimicrobial susceptibility testing, twentieth informational supplement, CLSI Document M100-S24, Wayne, Pennsylvania: CLSI 2014.

7. Das A, Ray P, Garg R, et al. (2001). Extended spectrum beta lactamase production in gram-negative isolates from cases of septicemia. In: Proceedings of Silver Jubilee Conference. New Delhi: All India Institutes of Medical Sciences; 21-25.

8. Borer A, Gilad J, Menashe G, et al. Extended spectrum betalactamase-producing Enterobacteriaceae strains in community acquired bacteremia in Southern Israel. Med Sci Monit. 2002 Jan; 8(1):CR44-7.

9. El-Karsh T, Tawfik AF, Al-Shammary F, et al. Antimicrobial resistance and prevalence of extended spectrum betalactamase among clinical isolates of gram-negative bacteria in Riyadh. J Chemother. 1995 Dec; 7(6):509-14.

10. AitMhand R, Soukri A, Moustaoui N, et al. Plasmidmediated TEM-3 extended-spectrum beta-lactamase production in Salmonella typhimurium in Casablanca. J Antimicrob Chemother. 2002 Jan; 49(1):169-72.

11. Shobha KL, Gowrish Rao S, Sugandhi Rao, et al. Prevalence of Extended Spectrum Beta-Lactamases in Urinary Isolates of Escherichia coli, Klebsiella and Citrobacter Species and their antimicrobial susceptibility pattern in a Tertiary Care Hospital. Indian Journal for the Practising Doctor Vol. 3, No. 6 (2007-01- 2007-02).
12. Baby Padmini S, Appalaraju B. Extended spectrum blactamases in urinary isolates of Escherichia coli and Klebsiella pneumoniae - prevalence and susceptibility pattern in a Tertiary Care Hospital Indian Journal of Medical Microbiology, (2004);22(3):172-174.

13. Mitali Chatterjee, Banerjee M, Guha S, et al. Prevalence of ESBL producing urinary isolates and their drug resistance pattern in Eastern part of India Sri Lankan Journal of Infectious Diseases Vol. 2(1) 2012:36-41.

14. Khurana S, Taneja N, Sharma M. Extended spectrum beta lactamase mediated resistance in urinary tract isolates of family Enterobacteriaceae. Indian J Med Res 2002; 116:145-9.

15. Mathur P, Kapil A, Das B, et al. Prevalence of extended spectrum beta lactamase producing gram negative bacteria in a Tertiary Care Hospital. Indian J Med Res 2002; 115:153-7.

16. Faisal Iqbal Afridi1, Badar Jahan Farooqi1 and Arif Hussain2. Frequency of extended spectrum beta lactamase producing Enterobacteriaceae among urinary pathogen isolates Journal of the College of Physicians and Surgeons Pakistan. 2011; Vol. 21(12):741-744.

17. Rahman MM, Haq JA (2004). Prevalence of extendedspectrum beta lactamase-producing Escherichia coli and Klebsiella pneumoniae in an urban hospital in Dhaka, Bangladesh. Int J Antimicrob Agents 24(5):508-10.

18. Feizabadi MM, Etemadi G (2006). Antibiotic-resistance patterns and frequency of extended-spectrum betalactamase-producing isolates of Klebsiella pneumoniae in Tehran. Med Sci Monit 12(11): BR362-5.

19. Jain A, Mondal R (2008). Detection of extended spectrum beta-lactamase production in clinical isolates of Klebsiella spp. Indian J Med Res 127(4):344-6.

20. Gold HS, Moellering RC Jr (1996). Antimicrobial-drug resistance. N Engl J Med 335(19):1445-53.

21. Ram S, Gupta R (2000). Emerging antibiotic resistance among the uropathogens. Indian J Med Sci 54(9):388-94.

22. Supriya S Tankhiwale, Suresh V Jalgaonkar, Sarfraz Ahamad, et al. Evaluation of extended spectrum beta lactamase in urinary isolates. Indian J Med Res 120, December 2004, pp 553-556.

23. Subha A, Ananthan S. Extended Spectrum Beta Lactamase (ESBL) mediated resistance to third generation cephalosporins among Klebsiella pneumoniae in Chennai. Indian J Med Microbiol 2002; 20:92-5.

24. Duttaroy B, Mehta S. Extended Spectrum B-Lactamases (ESBL) in clinical isolates of Klebsiella pneumoniae and Escherichia coli. Indian J Pathol Microbiol. 2005; 48:45-8.

25. Dr. Anbumani Narayanaswamy* $\Psi$ MD PhD and Prof M Mallika** MD. Prevalence and susceptibility of extended spectrum beta-lactamases in urinary isolates of Escherichia coli in a Tertiary Care Hospital, Chennai-South India. Internet Journal of Medical Update 2011 January;6(1):39-43. 


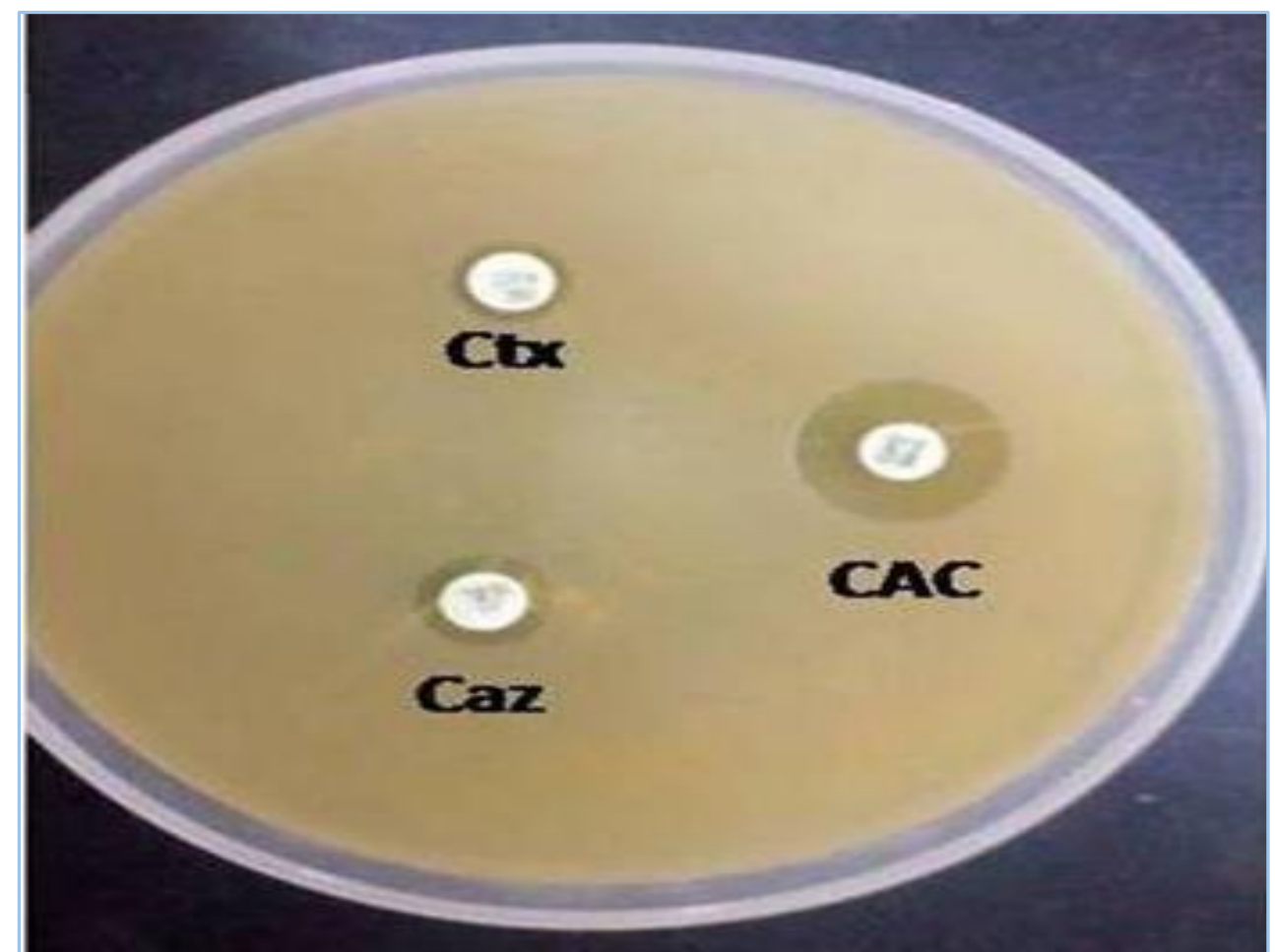

Fig. 1: Screen test positive isolate showing: Positive DDST test when swabbed on MH agar and incubated with Ceftazidime (Caz), Cefoaxime (Ctx) and Ceftazidime-Clvulanate (CAC)

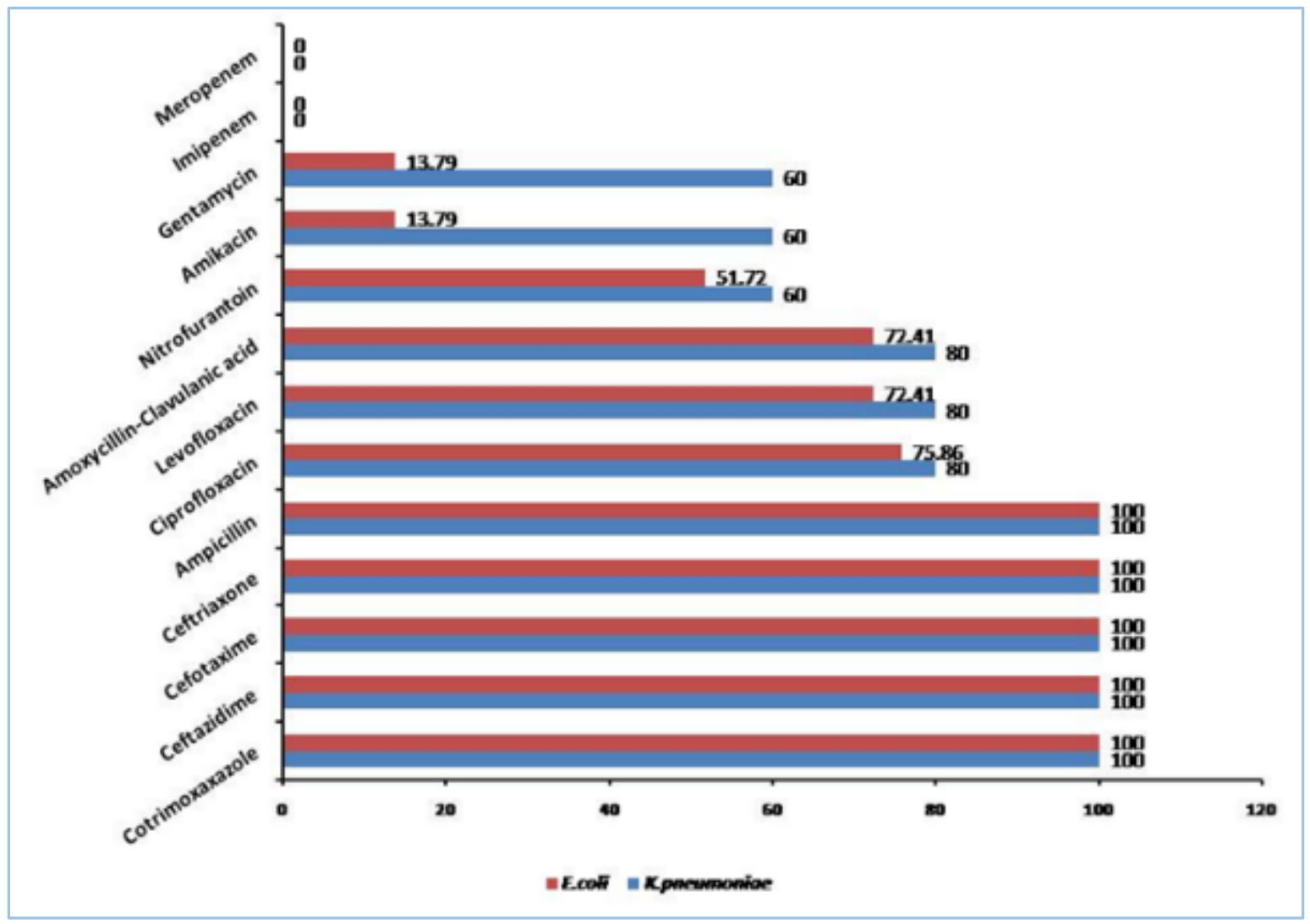

Fig. 2: Percentage of resistance to various Antibiotics of ESBL producers 